\title{
A perfect adaptation
}

The inability of a species of Candida to synthesize a common vitamin is one secret behind its success as a urinary pathogen, according to a new report in Science.

Candida glabrata is a common cause of urinary tract infections and adheres to host uroepithelial cells using adhesins that are encoded by the EPA gene family. The EPA genes are located in the subtelomeric regions of the C. glabrata genome - chromosomal locations that are transcriptionally silent, in part owing to the formation of repressive chromatin by the Sir complex of histone deacetylases. The EPA gene products aid colonization of the host, and so the Cormack laboratory and colleagues set out to understand the intricacies of chromatin-based regulation of C. glabrata adherence.

The authors used an elegant genetic approach to generate a $C$. glabrata reporter strain that monitors the transcriptional activity of the EPA6 locus. In contrast to mice with bloodstream infections - in which the reporter construct was transcribed at low levels - in a mouse model of urinary tract infection, reporter construct expression increased markedly. It seemed that, at least for C. glabrata, the mouse urinary tract was somehow able to break the silence imposed by the Sir proteins and to induce the expression of EPA6. By measuring the expression of the EPA6 gene in specific synthetic urine media, the authors established that the key to this phenomenon was the low levels of the vitamin nicotinic acid (NA) in urinary secretions.

But how can the lack of NA affect chromatin-mediated gene regulation? By comparative genome analysis, it became apparent that C. glabrata has deleted the genes that are needed to synthesize NA de novo, and therefore the organism is totally reliant on its surroundings to gain access to this vitamin. The authors proposed that, as NA is a precursor of $\mathrm{NAD}^{+}$, the $\mathrm{NAD}^{+}$-dependent histone-deacetylase activity of the Sir proteins might be reduced when NA is limiting. Consistent with a general loss of Sir-mediated silencing, when C. glabrata was grown in urine, not only EPA6, but two other silenced

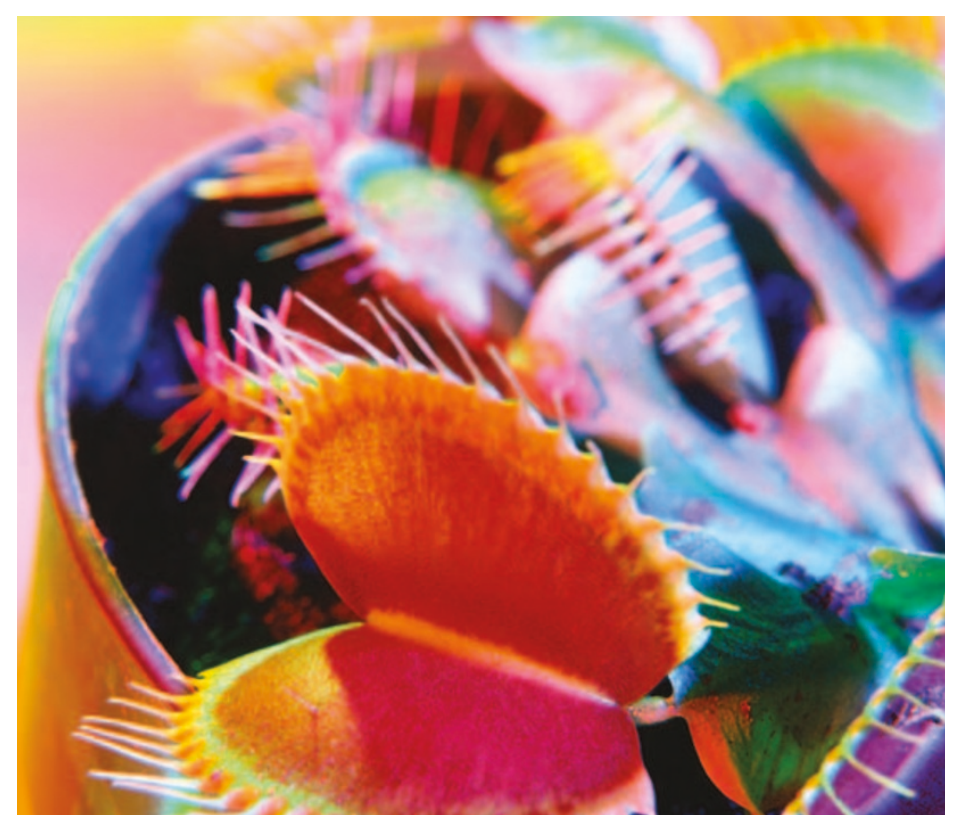

subtelomeric genes were derepressed, and the addition of excess NA suppressed this induction.

The authors suggest that the combined loss of the NA biosynthetic genes, together with the subtelomeric location of the EPA adhesin locus, represent an ingenious evolutionary strategy that ensures that C. glabrata is perfectly adapted to its ecological niche.

Shannon Amoils

(2) References and links

ORIGinAL RESEARCH PAPER Domergue, $R$.

et al. Nicotinic acid limitation regulates silencing of Candida adhesins during UTI. Science $17 \mathrm{Mar}$ 2005 (doi:10.1126/science.1108640) 